\title{
The role of observer strategy in the single-target $A B$ paradigm
}

\author{
Hayley E. P. Lagroix • Thomas M. Spalek • \\ Vincent Di Lollo
}

Published online: 6 July 2011

(C) Psychonomic Society, Inc. 2011

\begin{abstract}
Identification of the second of two targets (T1, T2) inserted in a stream of distractors is impaired when presented 200-500 ms after the first (attentional blink, AB). An AB-like effect has been reported by Nieuwenstein, Potter, and Theeuwes, Journal of Experimental Psychology: Human Perception and Performance, 35, 159-169, (2009, Experiment 2), with a distractor stream that contained only one target and a gap just before the target. Nieuwenstein et al. hypothesized that the gap enhanced the salience of the last distractor, causing it to be processed much like $\mathrm{T} 1$ in conventional AB studies. This hypothesis can also account for Lag-1 sparing (enhanced target performance when presented directly after the last distractor, without an intervening gap). We propose an alternative account of the Lag-1 sparing in the single-target paradigm based on observer strategy, and test it by presenting the single-target and dualtarget conditions to separate groups (Experiment 2) instead of mixed across trials (Experiment 1 and Nieuwenstein et al.'s study). The single-target condition exhibited Lag-1 sparing when it was mixed with the dual-target condition, but Lag-1 deficit when it was done in isolation. This outcome is consistent with an observer-strategy account but not with an enhanced salience account of the Lag-1 sparing effect in the single-target condition.
\end{abstract}

H. E. P. Lagroix $\cdot$ T. M. Spalek $\cdot$ V. Di Lollo

Simon Fraser University,

Burnaby, British Columbia, Canada

T. M. Spalek $(\bowtie)$

Department of Psychology, Simon Fraser University,

8888 University Drive,

Burnaby, BC V5A 1S6, Canada

e-mail: tspalek@sfu.ca
Keywords Attention · attentional blink - attention and executive control $\cdot$ attention capture

There is a cost to distributing attention to more than one task. As a rule, tasks are performed more slowly and less accurately when done concurrently than when done singly. This cost is in evidence not only when two tasks are performed concurrently, but also when they are performed in close temporal succession. A well-known example is the phenomenon known as attentional blink (AB): When two visual targets are inserted in a stream of distractors displayed in rapid serial visual presentation (RSVP), identification accuracy is nearly perfect for the first (T1), but is dramatically reduced for the second (T2; Raymond, Shapiro, \& Arnell, 1992). The AB is most pronounced when the temporal lag between the two targets is less than about $500 \mathrm{~ms}$.

Typically, distractor items continue to be presented during the intertarget lag. However, Nieuwenstein, Potter, and Theeuwes (2009) have recently reported that a substantial $\mathrm{AB}$ occurs even when the intervening distractors are replaced by blank frames - namely, by a gap in the RSVP stream, as illustrated in the dual-target sequence in Fig. 1. A related finding was that an $\mathrm{AB}$-like deficit occurred even when $\mathrm{T} 1$ was replaced by a distractor, as illustrated in the single-target sequence in Fig. 1. The findings of Nieuwenstein et al. (2009, Experiment 2) are illustrated in the right half of Fig. 1, redrawn from their Fig. 3. The present work is concerned exclusively with the single-target results illustrated in Fig. 1 (open symbols). To be sure, dual-target conditions were also included in the present experiments, but their function was strictly ancillary.

In addition to the AB-like effect (impaired accuracy of T2 identification at Lag 3 relative to Lag 7), the singletarget results in Fig. 1 exhibit a Lag-1-sparing-like effect 

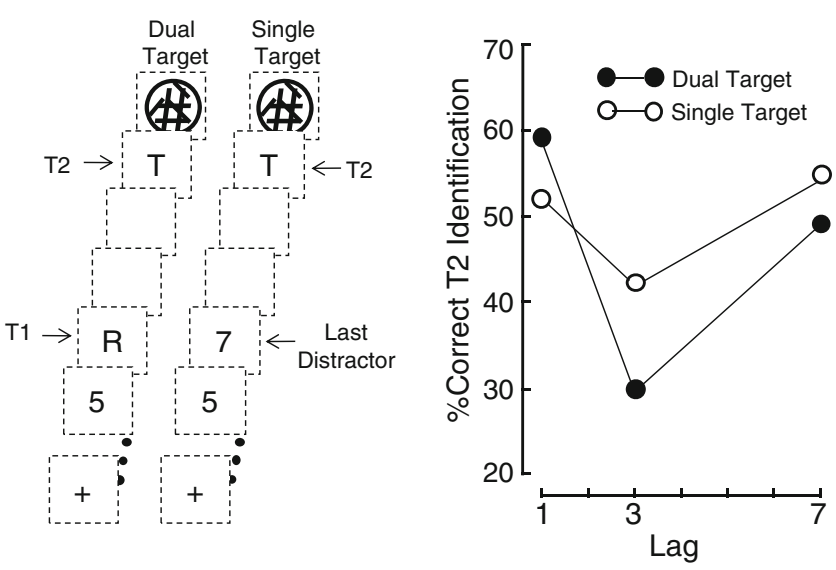

Fig. 1 Left-hand panel: Schematic representation of the sequence of events in the dual-target and single-target conditions at Lag 3. Righthand panel: Results of Nieuwenstein et al. (2009, Experiment 2), redrawn from their Fig. 3. T1 first target; T2 second target

(enhanced accuracy of T2 identification at Lag 1 relative to Lag 3). ${ }^{1}$ Nieuwenstein et al. (2009) accounted for both effects in terms of enhanced salience of the last distractor when it was directly followed by a gap, as explained below. Here, we consider an alternative possibility that both effects in the single-target condition may have arisen from observer strategy.

Nieuwenstein et al. (2009) accounted for the AB in the single-target condition (Fig. 1, open symbols) on the hypothesis that the last distractor was followed directly not by a target that acted as a backward mask but by a blank gap in the RSVP stream. The absence of masking caused the salience of the last distractor to be enhanced. Because of its enhanced salience, the distractor captured attention and was processed much like $\mathrm{T} 1$ in the dual-target condition, leading to an AB. Although Nieuwenstein et al. did not offer an explicit account of the Lag-1 sparing obtained in the single-target condition, an account can be given in terms of their enhanced-salience hypothesis of the AB. Because at Lag 1 in the single-target condition the last distractor was not followed by a gap, its salience was not enhanced and, therefore, it did not capture attention. This allowed attention

\footnotetext{
${ }^{1}$ A word about terminology is in order. In conventional AB studies, the term lag denotes the position of $\mathrm{T} 2$ relative to $\mathrm{T} 1$ in the RSVP stream. Thus, at Lag 1, T2 comes directly after T1, and at Lag 3, it is in the third position after T1. In the single-target condition, following the practice of Nieuwenstein et al. (2009), we use the term lag to denote the position of the only target relative to the last distractor in the RSVP stream. For example, in the single-target sequence illustrated in Fig. 1, the target is presented at Lag 3. Also, to maintain comparability with the practice of Nieuwenstein et al., we use the term $T 2$ to denote not only the second target in the dual-target condition, but also the only target in the single-target condition. Similarly, we use the term Lag-1 sparing (the finding that $\mathrm{T} 2$ escapes the $\mathrm{AB}$ when presented directly after T1) not only in the dual-target condition but also in the single-target condition, even though in that condition, the target is preceded not by another target but by a distractor.
}

to be deployed to the target more readily at Lag 1 than at Lag 3 at which the intervening gap allowed the last distractor to capture attention to the detriment of the target. Thus, both the AB and the Lag-1 sparing effects in the single-target condition are explained in terms of a single mechanism: enhancement (or lack thereof) of the last distractor.

In the present study, we consider the possibility that the Lag-1 sparing effect in the single-target condition (Fig. 1, open symbols) may have been due, at least in part, to observer strategy. An important procedural detail in the study of Nieuwenstein et al. (2009, Experiment 2) was that single-target and dual-target trials were mixed randomly so that on any given trial, observers could not anticipate whether the RSVP stream contained one target or two. Under these conditions, the leading stream of distractors was followed directly by a target on the majority of trials (T1 in all six lags used by Nieuwenstein et al. in the dualtarget condition, T2 at Lag 1 in the single-target condition). This means that the gap in the RSVP stream could not be used as a reliable cue for the first (or the only) target because it preceded that target on only five out of 12 trials. The optimal strategy, in this case, would be to monitor the RSVP stream for the occurrence of a target at the end of the leading stream of distractors. This strategy would enhance identification accuracy for $\mathrm{T} 1$ in the dual-target condition and for the only target (T2) at Lag 1 in the single-target condition, resulting in Lag-1 sparing in the single-target condition.

Predictions from the observer-strategy hypothesis of Lag-1 sparing can be tested against the corresponding predictions from the enhanced-salience hypothesis by adopting a between-subjects design in which the singletarget task is performed by one group of observers and the dual-target task by another group. According to the observer-strategy hypothesis, the optimal strategy for the single-target group would be to look not for a target, as in the Nieuwenstein et al. (2009) experiment in which singletarget and dual-target trials were mixed, but for a gap in the RSVP stream as a cue for the upcoming target. This is because on the majority of trials (i.e., at all lags other than Lag 1) the target would be preceded directly by a gap. While enhancing identification accuracy at lags in which the target is preceded by a gap, however, this strategy would lead to a deficit at Lag 1 because the observer's expectation would be violated by the occurrence of a target instead of the expected gap.

According to the enhanced-salience hypothesis, on the other hand, Lag-1 sparing should be in evidence in the single-target group, just as it is when the trials are mixed (Fig. 1, open symbols). This is because, in both cases, the last distractor is followed not by a gap but by the target; hence, its salience is not enhanced in either case. As noted 
above, lack of distractor enhancement at Lag 1-coupled with gap-triggered enhancement at Lag 3-should lead to Lag-1 sparing.

In the present work, we tested the enhanced-salience and the observer-strategy hypotheses by presenting the singletarget and the dual-target conditions either to separate groups of observers or to a single group with the conditions mixed randomly across trials. On the enhanced-salience hypothesis, Lag-1 sparing should be in evidence in the single-target condition when single-target and dual-target trials are presented to separate groups, much as it was when the trials were mixed (Fig. 1, open symbols). In contrast, according to the observer-strategy hypothesis, blocking the conditions should produce a Lag-1 deficit.

In pilot runs, we found that identification accuracy was constrained by a response ceiling imposed by the upper limit of the $100 \%$ response scale. We avoided that ceiling in the present experiments by using a dynamic threshold tracking procedure known as parameter estimation by sequential testing(PEST; Taylor \& Creelman, 1967), which is described below and is inherently free from response constraints. Having opted for this technique, an essential first step was to verify that the results reported by Nieuwenstein et al. (2009, Experiment 2) could be replicated with the PEST procedure. This was done in Experiment 1.

\section{Experiment 1}

\section{Method}

Participants Forty-seven undergraduate students participated for course credit. All reported normal or corrected-to normal vision and were naive to the purpose of the experiment. In this and all subsequent experiments, participants gave written informed consent prior to the experiment. The Office of Research Ethics at Simon Fraser University approved this study.

Apparatus and stimuli Stimuli were displayed on an NEC AccuSync CRT monitor with a resolution of $800 \times 600$ pixels at a refresh rate of $140 \mathrm{~Hz}$. All stimuli were black (20-point Helvetica font; RGB 0, 0, 0;0.9 cd/m²) and were presented in the center of the screen against a gray background (RGB 90, 90, 90; $15.0 \mathrm{~cd} / \mathrm{m}^{2}$ ). The RSVP stream contained a variable number of digit distractors (2-9) and, depending on the target condition, either one or two uppercase letter targets (T1 and T2) selected randomly without replacement from the English alphabet, except I, O, W, and M. All subtended approximately 0.6 degrees vertically. Following Nieuwenstein et al. (2009), T2 was followed by a pattern mask consisting of a white square in which a circle, pound sign, and three additional line segments were drawn in black (see Fig. 1). The experiment was programmed in E-Prime (Version 2.0; Psychological Software Tools, Pittsburgh, PA).

Design and procedure The experiment was run in a dimly lit room. At the beginning of the session, participants were required to read the instructions displayed on the screen, and were invited to ask questions. All displays were viewed from a distance of approximately $60 \mathrm{~cm}$. At the beginning of each trial, a small fixation cross was presented in the center of the screen. Participants initiated each trial by pressing the space bar, at which point the fixation cross disappeared and the RSVP sequence began.

In the dual-target condition, the number of distractors preceding $\mathrm{T} 1$ was determined randomly on each trial and varied between five and eight, inclusive. In the single-target condition, an additional distractor was displayed in the place of T1, so that between six and nine distractors were displayed. On any given trial, the distractors were selected randomly, with replacement, from the set of digits 2-9, with the constraint that the selected digit was not one of the two preceding items. With the exception of the mask, each item in the RSVP stream remained on the screen for approximately $7 \mathrm{~ms}$. T1 and all distractors were followed by an interstimulus interval (ISI) of approximately $93 \mathrm{~ms}$ during which the screen remained blank, yielding a presentation rate of 10 items/s. For Lags 3 and 7 in the dual-target condition, an additional blank period was inserted between $\mathrm{T} 1$ and T2. The duration of the additional gap was $200 \mathrm{~ms}$ at Lag 3 and $600 \mathrm{~ms}$ at Lag 7 (see Fig. 1). In the singletarget condition, corresponding additional gaps of 200 and $600 \mathrm{~ms}$ were inserted between the last distractor and T2 (see Fig. 1). The ISI between $\mathrm{T} 2$ and the mask was governed by PEST to yield the critical ISI (ISI $\mathrm{C}_{\mathrm{C}}$, see below) at which T2 could be identified on approximately $80 \%$ of the trials. The pattern mask for T2 was presented for $400 \mathrm{~ms}$. Examples of the stimuli and the sequence of events on any given trial are illustrated in Fig. 1.

At the end of each trial, the number of targets presented on that trial was displayed on the screen, and the participants were prompted to identify the target(s) by pressing the corresponding key(s) on the keyboard. They were instructed to report the letters in any order, and they were allowed to guess if uncertain. The fixation cross then reappeared, indicating readiness for the next trial.

As noted above, the dependent measure was the $\mathrm{ISI}_{\mathrm{c}}$ between $\mathrm{T} 2$ and its trailing mask that yielded $80 \%$ correct $\mathrm{T} 2$ responses. The ISI between $\mathrm{T} 2$ and the trailing mask was varied dynamically by a threshold-tracking staircase procedure (PEST), which reduced the ISI when the observer's response accuracy exceeded the criterial level, and it increased the ISI when accuracy was too low. A Wald 
(1947) sequential likelihood-ratio test determined whether the accuracy of the immediately preceding run of responses was greater or less than $80 \%$. In the dual-target condition, the Wald routine was called only on trials in which the first target had been identified correctly.

Each participant took part in one session, consisting of two blocks. In each of the two blocks, there were six independent and randomly intermixed PEST runs, one for each of the three lags in the dual-target and the single-target conditions. The assumption was made that the criterial level of performance had been approximated after three reversals in the direction of adjustment of the ISI. The final score $\left(\right.$ ISI $\left._{c}\right)$ was the mean T2-mask ISI over the last 12 trials following the third reversal, separately for each of the six combinations of lag and target condition.

\section{Results and discussion}

Accuracy of T1 identification in the dual-target condition was $86 \%, 97 \%$, and $95 \%$ for Lags 1, 3, and 7, respectively. Planned comparisons revealed that Lag 1 differed significantly from Lag 3, $t(46)=3.62, p=.001$, and from Lag $7, t(46)=$ $2.17, p=.035$, which did not differ significantly from one another $(t<1)$. Averaged across blocks and over the 12 trials following the third reversal, the values of ISI $_{c}$, averaged across observers separately for each lag and target condition, are illustrated in Fig. 2. The data were analyzed in a 2 (target condition: single or dual) x 3 (lag: 1, 3, or 7) repeated measures ANOVA. The analysis revealed significant effects of target condition, $F(1,46)=181.21$, $M S_{\mathrm{e}}=34.03, p<.001$, partial $\eta^{2}=.798$, and lag, $F(2,92)=$ $88.83, M S_{\mathrm{e}}=40.73, p<.001$, partial $\eta^{2}=.659$. The interaction effect was also significant, $F(2,92)=47.07$, $M S_{\mathrm{e}}=25.96, p<.001$, partial $\eta^{2}=.506$. The interaction effect was explored with two additional ANOVAs. One was aimed at testing the magnitude of Lag 1 sparing and consisted of a 2 (target condition: single or dual) x 2 (lag:

Fig. 2 Results of Experiment 1 in which single-target and dualtarget displays were mixed randomly across trials. $I S I_{C}$ critical ISI for $80 \%$-correct identification of the second (or the only) target; ISI interstimulus interval; $m s$ milliseconds. Error bars indicate standard errors

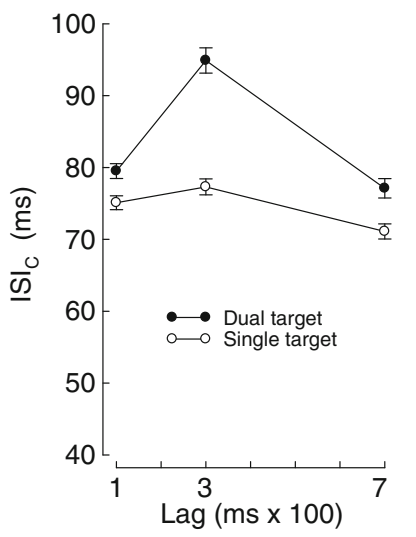

1 or 3 ) repeated measures ANOVA. This analysis yielded a significant interaction effect indicating that the magnitude of Lag-1 sparing was greater in the dual-target than in the single-target condition, $F(1,46)=100.20, M S_{\mathrm{e}}=20.41, p<$ .001 , partial $\eta^{2}=.685$. The second analysis was aimed at testing the magnitude of the $\mathrm{AB}$ and consisted of a 2 (target condition: single or dual) x 2 (lag: 3 or 7) repeated measures ANOVA. This analysis yielded a significant interaction effect indicating that the magnitude of the $\mathrm{AB}$ was greater in the dual-target than in the single-target condition, $F(1,46)=$ 57.73, $M S_{\mathrm{e}}=27.58, p<.001$, partial $\eta^{2}=.557$. Planned comparisons between the means for Lags 1 and 3 indicated that significant Lag-1 sparing was obtained in both the dualtarget, $\mathrm{t}(46)=10.52, p<.001$, and in the single-target, $\mathrm{t}(46)=$ $2.46, p=.018$, conditions. Similar comparisons between Lags 3 and 7 confirmed that significant ABs were obtained in both the dual-target, $\mathrm{t}(46)=11.85, p<.001$, and in the single-target, $\mathrm{t}(46)=8.30, p<.001$ conditions.

The results in Fig. 2 are consistent with the results of Nieuwenstein et al. (2009, Experiment 2), illustrated in Fig. 1. It is important to note that because $\mathrm{ISI}_{\mathrm{C}}$ reflects the mask-free interval required to identify $\mathrm{T} 2$ to the criterial level, high T2 identification scores in Fig. 1 correspond to low ISI $_{C}$ scores in Fig. 2. Clearly, the results obtained by Nieuwenstein et al. with accuracy as the dependent measure were replicated in the present experiment with $\mathrm{ISI}_{\mathrm{C}}$. On the basis of this finding, ISI $_{C}$ was used as the dependent measure in Experiment 2.

Both Lag-1 sparing and the $\mathrm{AB}$ were in evidence in the single-target condition. This finding is consistent with both the enhanced-salience and the observer-strategy hypotheses outlined above. The two hypotheses are decoupled in Experiment 2.

\section{Experiment 2}

The principal objective of Experiment 2 was to test contrasting predictions from the enhanced-salience and the observer-strategy hypotheses regarding Lag-1 sparing. This was done by presenting the two conditions to different groups of observers. Predictions from the enhancedsalience hypothesis are straightforward. Given that the salience of the last distractor is unaffected by whether the conditions are mixed across trials or presented in isolation, Lag-1 sparing should be in evidence in either case. In contrast, predictions from the observer-strategy hypothesis hinge on the probability of a gap preceding the target in the RSVP stream. When that probability is high, observers will adopt a strategy of looking for the gap as a cue for the target. As noted in the introduction, this strategy would lead to a deficit at Lag 1 because the observer's expectation would be violated by the occurrence of a target instead of 
the expected gap. Given that the probability of a gap preceding the target is higher when the single-target condition is done in isolation, a Lag-1 deficit should be in evidence in the singletarget group in the present experiment as distinct from the Lag1 sparing in the corresponding condition in Experiment 1.

\section{Method}

Participants Twenty eight undergraduate students participated for course credit. They were allocated randomly to two groups of 14: the single-target group and the dual-target group. All reported normal or corrected-to normal vision and were naive to the purpose of the experiment. None had participated in Experiment 1.

Design and procedures Apparatus, design, and procedures were the same as in Experiment 1, with the exception that the dual-target and the single-target conditions were performed by separate groups of participants.

\section{Results and discussion}

Accuracy of $\mathrm{T} 1$ identification in the dual-target condition was $96 \%, 90 \%$, and $97 \%$ for Lags 1,3 , and 7 , respectively. Planned comparisons revealed no significant differences among the means (all $t \mathrm{~s}<1.03$ ). Averaged over the 12 trials following the third reversal, the values of $\mathrm{ISI}_{\mathrm{c}}$, averaged across observers, separately for each lag and group, are illustrated in Fig. 3. The data were analyzed in a 2 betweensubjects (group: single target or dual target) x 3 withinsubjects (lag: 1, 3, or 7) ANOVA. The analysis revealed significant effects of group, $F(1,26)=9.44, M S_{\mathrm{e}}=255.40$, $p=.005$, partial $\eta^{2}=.266$, and lag, $F(2,52)=36.62, M S_{\mathrm{e}}=$ $54.79, p<.001$, partial $\eta^{2}=.585$. The interaction effect was also significant, $F(2,52)=19.27, M S_{\mathrm{e}}=54.79, p<.001$, partial $\eta^{2}=.426$. Planned comparisons between the means for Lags 1 and 3 indicated that significant Lag-1 sparing

Fig. 3 Results of Experiment 2 in which single-target and dualtarget displays were presented separately to different groups of observers. $I S I_{C}$ critical ISI for $80 \%$ correct identification of the second (or the only) target; ISI interstimulus interval; $m s$ milliseconds. Error bars indicate standard errors was obtained in the dual-target group, $t(13)=4.89, p<.001$, and that a significant Lag-1 deficit was obtained in the single-target group, $t(13)=2.25, p=.042$. Similar comparisons between Lags 3 and 7 confirmed that significant ABs were obtained in both the dual-target, $t(13)=9.67$, $p<.001$, and the single-target, $t(13)=3.08, p=.009$, groups.

The outcome of interest in the present experiment is that a Lag-1 deficit was in evidence in the single-target condition (Fig. 3, open symbols) as distinct from the Lag-1 sparing obtained in the corresponding condition in Experiment 1 (Fig. 2, open symbols). This was confirmed by a 2 (Lags 1 and 3) $\times 2$ (Experiments 1 and 2) mixed ANOVA that revealed no significant effects of lag, $F(1,62)=3.42, M S_{\mathrm{e}}=33.86, p>$ .05 , but significant effects of experiment, $F(1,162)=16.25$, $M S_{\mathrm{e}}=206.62, p<.001$, partial $\eta^{2}=.208$, and a Lag $\mathrm{x}$ Experiment interaction, $F(1,162)=14.13, M S_{\mathrm{e}}=33.86, p<$ .001 , partial $\eta^{2}=.186$. This pattern of results is consistent with the idea, outlined previously, that observers adopt different strategies in performing the single-target task when it is mixed with the dual-target task than when it is presented in isolation. The theoretical implications of these results are considered below.

A glance at Figs. 2 and 3 reveals that performance was better (ISI ${ }_{C}$ was shorter) in Experiment 2 (Fig. 3) than in Experiment 1 (Fig. 2). In other words, performance was better when the single-target trials were done in isolation (Fig. 3) than when they were mixed with dual-target trials (Fig. 2). Notably, the improvement was not limited to the single-target condition but was also in evidence in the dualtarget condition. These outcomes strongly suggest that the task was harder in the mixed condition in which there was uncertainty as to the number of targets in the RSVP stream. The finding that uncertainty was an important factor is consistent with the idea that expectation played a role in these experiments.

\section{General discussion}

The present work was concerned mainly with the interpretation of the Lag-1-sparing effect obtained in single-target RSVP streams in which the target is preceded by a gap at all lags except Lag 1 (Nieuwenstein et al., 2009, Experiment 2). We began by replicating the findings of Nieuwenstein et al. using a threshold-tracking procedure that was not subject to ceiling constraints. In Experiment 1, dual-target and singletarget conditions were mixed randomly as was done by Nieuwenstein et al., whose results were replicated, including $\mathrm{AB}$ deficits and Lag-1 sparing in both conditions. In Experiment 2, the dual- and the single-target conditions were performed by separate groups of observers. The dualtarget results were similar to those obtained in Experiment 1. The results in the single-target condition, however, yielded a 
Lag-1 deficit, consistent with the hypothesis that performance was determined by observer strategy, but inconsistent with the enhanced-salience hypothesis.

It is possible that observer strategy may also have mediated the $\mathrm{AB}$ in the single-target condition in Experiment 2. On the hypothesis that observers were looking for a gap in the RSVP stream, the poorest performance should occur at Lag 1 , at which the target is not preceded by a gap. Performance at Lag 3 would also be somewhat impaired because, upon detecting the gap, observers would have to switch from gapdetection mode to target-processing mode, and the target would arrive before the switch could be accomplished. Performance would be best at Lag 7 because the $600-\mathrm{ms}$ blank period is sufficient for completing the switch, thus optimizing readiness for target identification. This hypothesis, however, is clearly ad hoc and in need of empirical verification.

Alternatively, it is possible that the observer-strategy hypothesis applies only to Lag-1 sparing and that the $\mathrm{AB}$ observed at Lag 3 in the single-target conditions in Experiments 1 and 2 was caused by enhanced salience of the last distractor, as proposed by Nieuwenstein et al. (2009). That hypothesis is buttressed by the findings of Spalek, Falcon, and Di Lollo (2006), who showed that a salient- - though task-irrelevant-item in the RSVP stream can produce an $\mathrm{AB}$. The hypothesis that Lag-1 sparing and the $\mathrm{AB}$ are mediated by distinct mechanisms is also consistent with the finding that Lag-1 sparing and the $\mathrm{AB}$ are believed to be independent events (Visser, Bischof, \& Di Lollo, 1999).
Both of the aforementioned hypotheses are plausible accounts of the AB. Only the observer strategy hypothesis, however, is capable of accounting for the Lag-1 deficit obtained in the single-target condition in Experiment 2.

Author Note This work was supported by an Alexander Graham Bell Canada Graduate Scholarship from the Natural Sciences and Engineering Research Council of Canada (NSERC) awarded to H.E.P. L., and by NSERC Discovery Grants awarded to T.M.S. and to V.D.L.

\section{References}

Nieuwenstein, M. R., Potter, M. C., \& Theeuwes, J. (2009). Unmasking the attentional blink. Journal of Experimental Psychology: Human Perception and Performance, 35, 159-169.

Raymond, J. E., Shapiro, K. L., \& Arnell, K. M. (1992). Temporary suppression of visual processing in an RSVP task: An attentional blink? Journal of Experimental Psychology: Human Perception and Performance, 18, 849-860.

Spalek, T. M., Falcon, L. J., \& Di Lollo, V. (2006). Attentional blink and attentional capture: Endogenous versus exogenous control over paying attention to two important events in close succession. Perception \& Psychophysics, 68, 674-684.

Taylor, M. M., \& Creelman, C. D. (1967). PEST: Efficient estimates on probability functions. Journal of the Acoustical Society of America, 41, 782-787.

Visser, T. A. W., Bischof, W. F., \& Di Lollo, V. (1999). Attentional switching in spatial and non-spatial domains: Evidence from the attentional blink. Psychological Bulletin, 125, 458-469.

Wald, A. (1947). Sequential Analysis. New York, NY: John Wiley \& Sons, Inc. 